\title{
Influence of Body-Surface Geometry Accuracy on Noninvasive Reconstruction of Electrical Activation and Recovery in Electrocardiographic Imaging
}

\author{
Matthijs JM Cluitmans ${ }^{1}$, Paul GA Volders ${ }^{1}$ \\ ${ }^{1}$ CARIM School for Cardiovascular Diseases, Maastricht University Medical Centre, Maastricht, the \\ Netherlands
}

\begin{abstract}
In Electrocardiographic Imaging (ECGI), electrical potentials on a patient's heart surface are noninvasively reconstructed from potential recordings on the body surface. This process depends on a geometrical description of the heart surface and body surface. Here, we investigate the influence of inaccuracies in the bodysurface geometry on ECGI reconstruction accuracy.

In four canines, $c a$. 200 electrodes were attached to the body surface and in total 93 beats recorded. A CT scan provided the exact position of these electrodes and the heart-surface. The body geometry was defined by the electrode positions only, and their position in the digital geometry was then varied: 1) for all electrodes collectively in the same direction, 2) or in a random direction (where electrodes on the same strip were taken together). Invasive recordings on the heart surface provided ground truth data to which noninvasive reconstructions with these varying geometries were compared, in terms of correlation coefficients between recorded and reconstructed potentials $(C C)$, localization error (LE) between known beat origin and reconstructed beat origin, and activation and recovery isochrones.

Results for displacement of all electrodes in a single direction or in random directions indicate that displacement up to $30 \mathrm{~mm}$ has a limited effect on ECGI accuracy. More importantly, consistent displacement of electrodes in one direction mainly results in a shift of the activation or recovery pattern in that direction. Patterns are also preserved when displacement is in random directions. Thus, inaccuracy in body-surface geometry up to $30 \mathrm{~mm}$ in ECGI preserves activation and recovery patterns but hampers their exact localization.
\end{abstract}

\section{Introduction}

The electrocardiogram (ECG) is a well-established, validated, patient-friendly, quick, reproducible and cheap tool to assess the electrical activation and recovery of the heart as projected on the body surface. However, it is a superimposed and 'smeared' representation of the actual cardiac electrical activity. Thus, it lacks the capacity to assess electrical activity at high resolution at the level of the heart muscle. Conversely, electrocardiographic imaging (ECGI) is a modality that noninvasively images electrical activation and recovery directly at the heart surface. ECGI computes direct representations of electrical activity at the heart surface by combining extensive recordings from $\sim 200$ body-surface electrodes with a precise, patient-specific torso-heart geometry. [3]

The patient-specific torso-heart geometry is usually segmented from a Computed Tomography (CT) scan or similar imaging modalities such as Magnetic Resonance Imaging (MRI). These modalities allow to determine the precise position of the electrodes on the body surface, and the exact shape and position of the heart surface. However, the need for CT or MRI complicates logistics in clinical settings, induces radiation burden (in case of CT), might be incompatible with implanted devices (in case of MRI), and often requires the use of contrast medium that is potentially nephrotoxic. Taken together, these issues make it difficult to apply ECGI repeatedly in the same patient (e.g., for follow-up).

In this study, we investigate the use of an approximate body-surface geometry and its influence on ECGI accuracy.

\section{Methods}

ECGI was performed in a canine experiment. The exact torso-heart geometry was obtained from a CT scan and then digitally manipulated to investigate the effect of inaccuracies in body-surface geometry on reconstruction accuracy of ECGI. Invasive data were obtained for validation purposes.

\subsection{In vivo recordings}

In vivo data were acquired in experiments with anesthetized dogs, which were approved by the institutional review committee for animal studies. In four normal anesthetized dogs, two silicone bands with 99 electrodes were implanted around the basal and mid-basal 


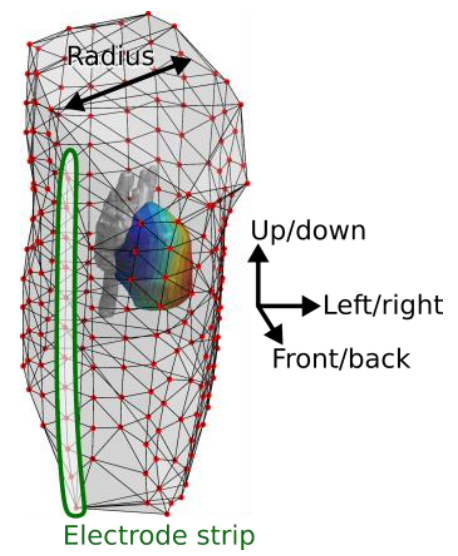

Figure 1. Torso-heart geometry in one of the canine experiments. Red dots indicate the body-surface electrodes, which fully define the body-surface geometry (gray triangles). The electrodes are arranged on strips, in this figure visible as columns (see green encircled region for one strip of electrodes). The digitized position of the body-surface electrodes is then changed in silico in varying directions and for varying amounts.

epicardium after thoracotomy. After chest closure, bodysurface electrodes (184 to 216 , depending on torso size) were attached to the chest. This number of body-surface electrodes is more than sufficient to obtain a good reconstruction with traditional methods. [2] Unipolar potential recordings were obtained simultaneously by the epicardial and body-surface electrodes. 93 beats were recorded during normal sinus rhythm or with pacing from the implanted electrodes.

\subsection{Inverse reconstruction}

The potential-based formulation of the forward/inverse problem of electrocardiography is based on the relation between potentials on a closed surface surrounding the heart, and the body surface. [3] The closed surface surrounding the heart is usually taken to be the epicardium (i.e., the outer heart surface) and was manually segmented, resulting in a mesh with approximately 1700 nodes. The body-surface can be a high-resolution mesh capturing the full shape of the torso, or consist of only the body-surface electrodes. Here, the body-surface geometry was fully defined by the electrodes only, i.e., the body-surface geometry consisted of 184-216 nodes depending in the number of used electrodes. The transfer matrix was then computed to capture the geometry and conductivity relation between the heart and body surfaces.

The goal of the inverse problem is to find the epicardial potentials from recorded body-surface potentials and the patient-specific transfer matrix. Regularization is necessary to handle the ill-posed nature of this problem.
We applied the well-known zeroth-order Tikhonov method, penalizing the potential amplitude.

From the resulting reconstructed epicardial potentials, we subsequently calculated the sequence of electrical activation and recovery (i.e., activation and recovery isochrones) on the heart surface, and the location of earliest activation (i.e., the origin of that beat). The reconstructed electrograms, activation isochrones, and recovery isochrones were then compared to the invasively recorded electrograms (correlation coefficient, CC), activation times $\left(\mathrm{R}_{\mathrm{act}}\right)$ and recovery times $\left(\mathrm{R}_{\mathrm{rec}}\right)$. Of the 93 recorded beats, 80 were paced (all from different locations). The known pacing location was compared to the reconstructed beat origin to compute the localization error (LE).

For full details on the in vivo experiments and the inverse reconstruction algorithms, we refer to our previous validation work. [1]

\subsection{Variation of torso geometry}

An example of the torso-heart geometry is shown in Figure 1. The position of the electrodes (i.e, nodes of the body surface) is varied in the digitized body-surface geometry in two different ways:

1. Changing the position of all electrodes simultaneously, in the same direction and with the same distance. (E.g., all electrodes moved upwards $20 \mathrm{~mm}$ with respect to the heart.)

2. Changing the position of all electrodes on a strip collectively, with a random direction and distance per strip. (E.g., all electrodes on strip 1 are moved $(20,-12,15) \mathrm{mm}$ in $(\mathrm{x}, \mathrm{y}, \mathrm{z})$ directions; and all electrodes on strip 2 are moved $(-12,18,-3) \mathrm{mm}$, etc.)

For case 1, the distance was varied with $+/-0,10,20$, 30,40 and $50 \mathrm{~mm}$ in either direction. For case 2, the distance was uniformly randomly varied up to $5,10,20$, 30,40 and $50 \mathrm{~mm}$. The possible directions of freedom were up/down, left/right and front/back movement, and radial decrease/increase (relative to the centre of the body).

For each new torso geometry, the transfer matrix was recalculated and the inverse reconstruction and evaluation were repeated.

\section{Results}

The results are shown in Figure 2 for electrogram morphology and beat origin localization, and in Table 1 for accuracy of activation and recovery times.

Radial decrease/increase of the body-surface geometry has relatively little influence on the reconstruction accuracy (Figure 2A). Even when the radius is increased by $50 \mathrm{~mm}$ (i.e., a $100 \mathrm{~mm}$ increase in diameter), correlation 


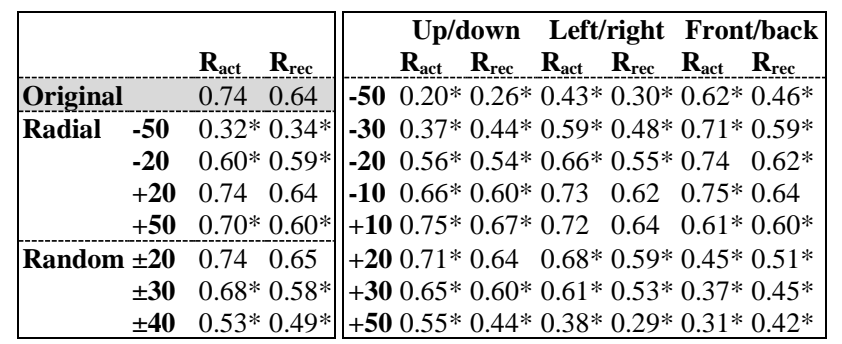

Table 1. Correlation coefficient between recorded and reconstructed activation times $\left(\mathrm{R}_{\mathrm{act}}\right)$ and recovery times $\left(\mathrm{R}_{\mathrm{rec}}\right)$, for varying body-surface geometries (displacement in $\mathrm{mm}$ ). Asterisk indicates statistical significant difference with original geometry.

coefficients are only marginally worse. Similarly, beat origin localization is not changing considerably either. For a decrease in radius, however, accuracy decreases more rapidly. This is possibly because the heart might lie outside the digitized torso when the torso geometry is shrunk too much. The results for accuracy of activation/recovery times are congruent (Table 2, category "radial").

For translational movement of all electrodes in the same direction (Figure 2B and Table 2, right), results are similar: only for large (>30 mm) displacement, accuracy decreases considerably. Again, one can see here that results mainly decrease in accuracy when the heart might penetrate the body surface due to too large displacement of body-surface electrodes. For example, as one can appreciate from Figure 1, backwards movement of the body geometry with $20 \mathrm{~mm}$ results likely in this situation, and can explain the sudden drop in accuracy that is visible in Figure 2B (green LE bar at $-20 \mathrm{~mm}$ ).

For random movement of all electrodes (direction and amount fixed per strip), results again indicate that there is little change in accuracy up to $30 \mathrm{~mm}$ displacement (Figure $2 \mathrm{C}$ and Table 2, bottom left).

Figure 3 shows the activation and recovery isochrones of an example beat. Up to $30 \mathrm{~mm}$ of random electrode displacement (panel B), these patterns are in general preserved and only shifted in location on the heart surface. Only for $40 \mathrm{~mm}$ displacement, the activation and recovery sequences cannot be recovered anymore. When the bodysurface geometry is translated as a whole, activation and recovery patterns move in the same direction over the heart surface. These general patterns remain preserved for up to $50 \mathrm{~mm}$ up/down displacement (panel C). Again, when displacement is likely resulting in (partial) positioning of the heart outside the body surface, accuracy is decreased significantly (see $50 \mathrm{~mm}$ example in panel D).

\section{Discussion}

In this study, we have examined the influence of accuracy of body-surface geometry on noninvasive reconstruction of electrical heart activity with ECGI.
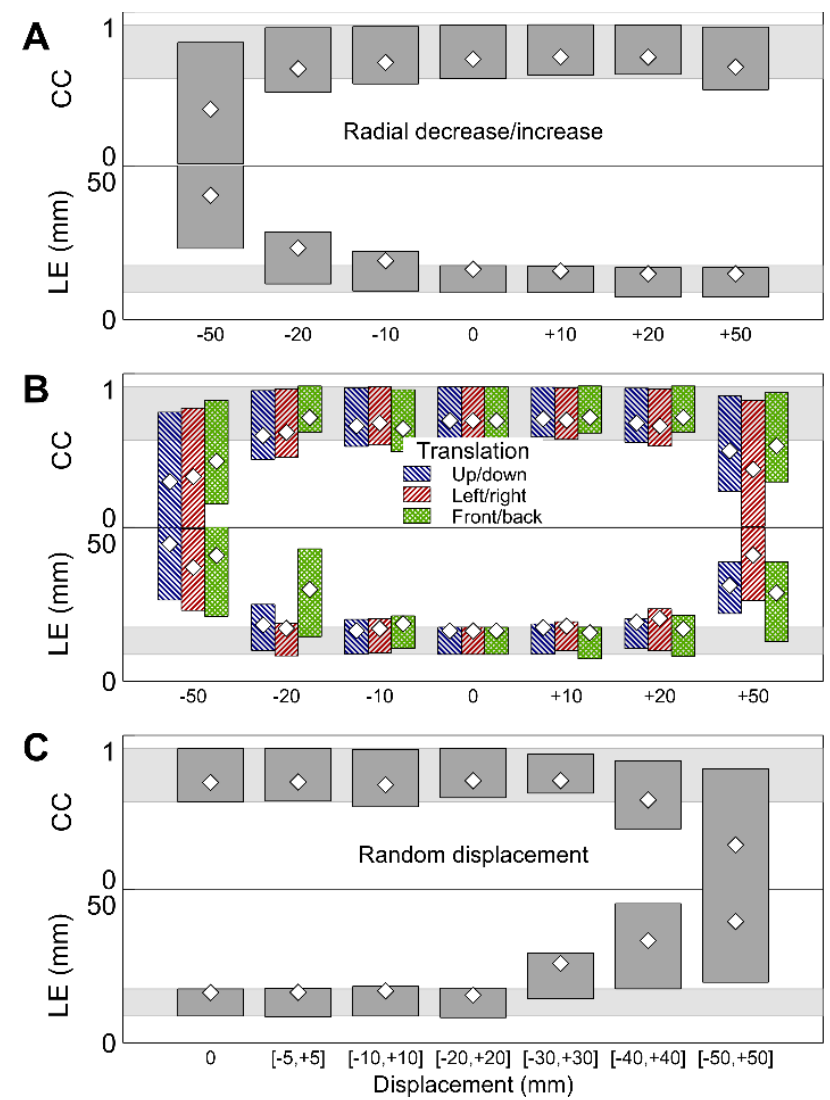

Figure 2. Correlation coefficient (CC) between invasively recorded and noninvasively ECGI-reconstructed electrograms, and localization error (LE) between known pacing location and reconstructed beat origin. Panel A: Results for torso geometries that were decreased and increased in radius. Panel B: Results for translational movement of the all electrodes collectively: up/down, left/right or front/back. Panel C: Results where all electrodes are translated in all directions for a random amount (all electrodes on the same strip taken together).

Overall, consistent displacement of electrodes (that form the body-surface geometry) in a certain direction results in a displacement of patterns on the heart surface in the same direction. For random displacements, results remain stable up to $20 \mathrm{~mm}$ in terms of beat origin localization, and up to $30 \mathrm{~mm}$ for electrogram morphology and activation/recovery time patterns.

More importantly, most severe drops in accuracy occur when the fixed heart geometry is likely (partially) outside the displaced torso geometry. As a dog's heart is only a few centimetres from the front and lateral sides of its torso, small displacements of those nearby electrodes can easily violate the heart-in-torso assumption. We did not exclude these cases from the analyses, but it is unlikely that such violating geometries are used in practice. In other words, 


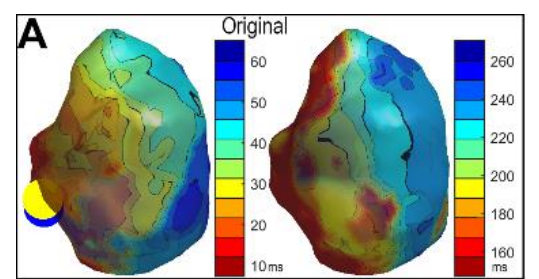

B

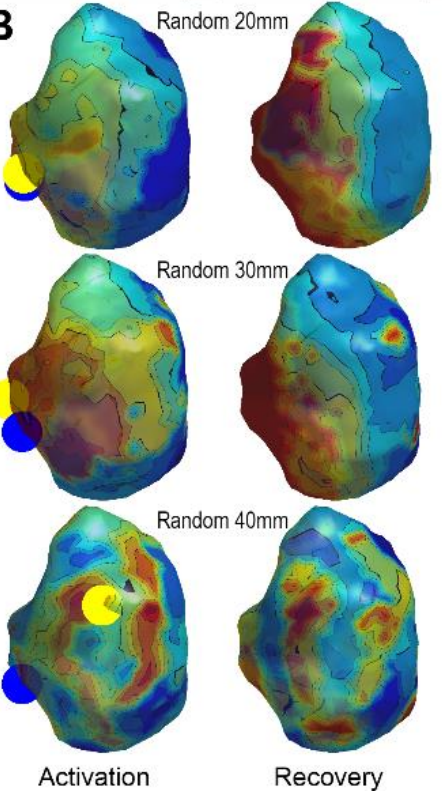

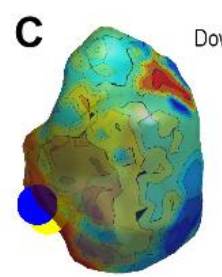

Down $20 \mathrm{~mm}$
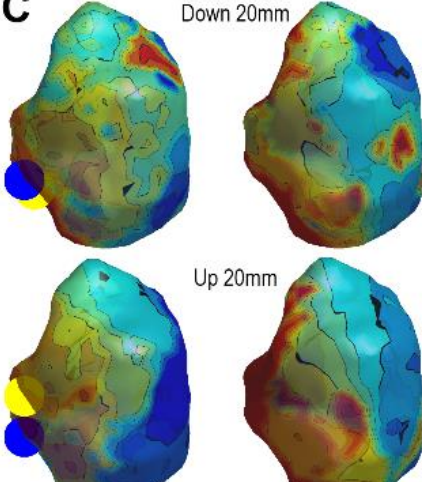

Up $20 \mathrm{~mm}$
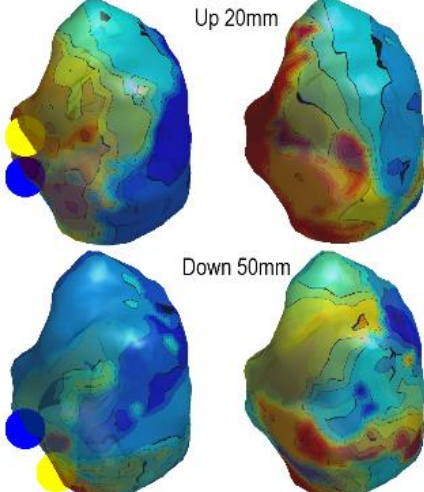

Down $50 \mathrm{~mm}$

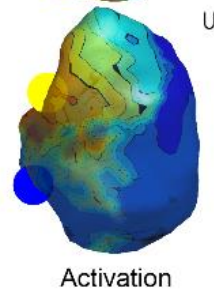

Recovery

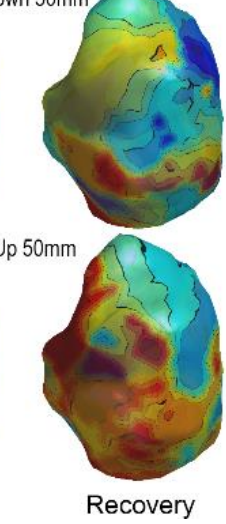

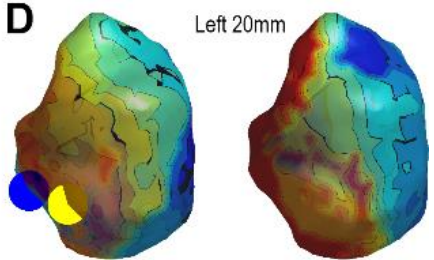

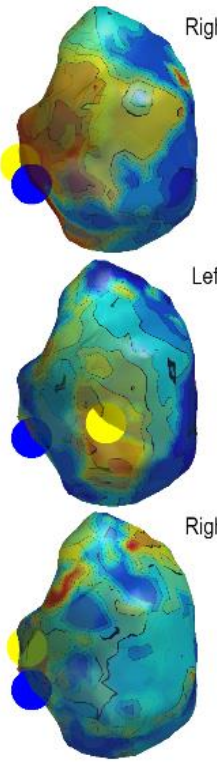

Activation
Right $20 \mathrm{~mm}$

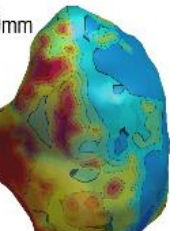

Figure 3. Activation and recovery isochrones on a canine heart for one beat. Blue dot: known pacing location; yellow dot: reconstruction location of earliest activation. Panel A: Isochrones as reconstructed with the default, exact torso geometry. Panel B: Results for random displacement of electrodes up to 20,30 or $40 \mathrm{~mm}$. Panel C: Isochrones for up/down movement of all electrodes consistently. Panel D: Isochrones for left/right movement of all electrodes consistently. Displacement of electrodes mainly results in displacement of the reconstructed isochrones in the same direction.

results for inaccurate torso geometries would likely be better than the worst-case scenarios we present here. Moreover, when interpreting the numbers in this study, one should take the difference in torso and heart sizes between canines and humans into account: the average human heart is 1.5 times bigger than canine hearts in this study. When extrapolated to humans, even larger displacements of electrodes could be acceptable.

In this study, we did not investigate the effect of heart displacement, as this problem is symmetric to torso displacement. Nevertheless, inaccuracies in the shape of the heart would create a non-symmetric problem and require further analysis.

In conclusion, this study shows through an in vivo analysis that inaccuracy in body-surface geometry up to 30 $\mathrm{mm}$ in ECGI preserves general activation and recovery patterns and electrogram morphology in canines, but hampers their exact localization. Thus, for the detection of abnormalities, an approximate torso geometry might be sufficient, whereas the localization of such abnormalities requires a torso geometry that is accurate $(\sim 20 \mathrm{~mm}$ in canines). It is likely that higher inaccuracies are acceptable in human-sized geometries.

\section{Conflicts of interest}

Matthijs Cluitmans is part-time employed by Philips Research. Philips Research is not involved in the study described in this paper and there are no conflicts of interest.

\section{References}

[1] M J M. Cluitmans, P. Bonizzi, J M H. Karel, M. Das, B L J H. Kietselaer, M M J. De Jong, F W. Prinzen, R L M. Peeters, R L. Westra, and P G A. Volders. In vivo validation of electrocardiographic imaging. 2017.

[2] Matthijs J M Cluitmans, J Karel, P Bonizzi, M de Jong, P Volders, R Peeters, and R L. Westra. In-vivo evaluation of reduced-lead-systems in noninvasive reconstruction and localization of cardiac electrical activity. In Computing in Cardiology, volume 42, pages 221-4, 2015.

[3] Matthijs J M. Cluitmans, R L M. Peeters, R L. Westra, and P G A. Volders. Noninvasive reconstruction of cardiac electrical activity: update on current methods, applications and challenges. Neth Heart J, 23(6):301-11, Jun 2015.

Address for correspondence: MJM Cluitmans, CARIM, Maastricht University, P.O. Box 616, 6200MD Maastricht, The Netherlands,m.cluitmans@maastrichtuniversity.nl. 